\title{
Dental management of patients with endocrine disorders
}

\author{
Laura Carlos Fabue $1^{1}$, Yolanda Jiménez Soriano ${ }^{2}, M^{a}$ Gracia Sarrión Pérez ${ }^{1}$ \\ ${ }^{1}$ Dentist. \\ ${ }^{2}$ Assistant Professor Doctor. Department of Stomatology. Medicine and dentistry faculty. Valencia University
}

Correspondence:

C/Tenor Garcia Romero, nro 5 pta 21

46015, Valencia

lauracarlosf@gmail.com

Carlos Fabue L, Jiménez Soriano Y, Sarrión Pérez MG. Dental management of patients with endocrine disorders. J Clin Exp Dent. 2010;2(4):e196-203.

http://www.medicinaoral.com/odo/volumenes/v2i4/jcedv2i4p196.pdf

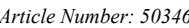

eMail: jced@jced.es
e Medina Oral L. L.

\begin{abstract}
Introduction: The endocrine system is responsible for hormonal secretion and is closely related to the central nervous system, as it diversifies its functions through the hypothalamus and pituitary glands. It controls physiological processes and maintains homeostasis. The neuroendocrine system is responsible for adaptation to environmental changes. Therefore, in dentistry, it is important to be aware of the risks and difficulties that may arise during the dental management of patients with endocrine disorders, and that visits to the dental clinics often represent a stressful situation.

Objectives: To review the literature on oral manifestations and dental management in patients with endocrine disorders (disorders of the thyroid, parathyroid and adrenal glands).

Material and Methods: For the literature review, we carried out a search in Pubmed / Medline database using limits and keywords according to the controlled vocabulary "Medical Subject Headings" (MeSH). We obtained a total of 19 articles (ten literature reviews, one case-control study, and eight clinical case series).

Results and discussion: We describe the most common oral manifestations in patients with endocrine disorders and the special dental management that should be carried out on these patients.
\end{abstract}

Key words: dental management, endocrine disorders, oral manifestations 


\section{Introduction}

The endocrine system is responsible for hormonal secretion and is closely related to the central nervous system, as it diversifies its functions through the hypothalamus and pituitary. It controls physiological processes and maintains homeostasis. The neuroendocrine system is responsible for adaptation to environmental changes. Also, a function of the nervous system is to provide a correct organic response. Its response may be primary, with the release of neurotransmitters, or if the stimulus prevails, the endocrine system secretes hormones. This is especially important in dentistry because many of the patients attending the dental clinics face stressful situations (1). Awareness is therefore necessary of the risks and difficulties that may arise during the dental management of patients with endocrine disorders and the most common oral manifestations.

\section{Objetives}

The aim of this article was to perform a literature review about:

1. Oral manifestations in patients with endocrine disorders (disorders of the thyroid, parathyroid and adrenal glands).

2. Dental management of patients with endocrine disorders (disorders of the thyroid, parathyroid and adrenal glands).

\section{Materials and methods}

For the literature review, we carried out a literature search in Pubmed / Medline database using the following words according to the controlled vocabulary (MeSH): "dental management", "oral manifestations", "hyperthyroidism", "hypothyroidism", "hyperparathyroidism" "primary hyperparathyroidism", "secondary hyperparathyroidism", "hypoparathyroidism", "Addison's disease", "primary adrenocortical insufficiency", "Cushing's syndrome", "adrenocortical hyperfunction". The limits used for the search were: articles in English or Spanish and articles published within the last 10 years. We reviewed a total of 19 articles of which ten were literature reviews, one case-control study and eight clinical case series.

\section{Results}

Oral manifestations and dental management of patients with thyroid gland disorders

The thyroid gland secretes three hormones: thyroxine (T4), triiodothyronine (T3) and calcitonin. T4 and T3 are hormones that affect metabolic processes throughout the body and are involved in oxygen use. Thyroidstimulating hormone (TSH or thyrotropin), produced by the pituitary gland, regulates the secretion of thyroid hormones (T4 and T3) through a negative feedback mechanism. Calcitonin is involved, with parathyroid hormone and vitamin $\mathrm{D}$, in regulating serum calcium and phosphorus levels and in the skeletal remodeling. Thyroid hormones influence the growth and maturation of tissues, energy metabolism and turnover of both cells and nutrients $(2,3)$.

- Hyperthyroidism

Hyperthyroidism or thyrotoxicosis is defined by a decrease in thyroid hormone production and thyroid gland function. It is caused by ectopic thyroid tissue, toxic thyroid adenoma, toxic multinodular goiter, subacute thyroiditis, factitious thyrotoxicosis and Graves' disease and diffuse toxic goiter, being the most common cause of hyperthyroidism $(2,4)$.

Hyperthyroidism can have clinical manifestations at gastrointestinal levels (weight loss, increased appetite, nausea and vomiting), in hair, skin and nails (thin and brittle hair, soft nails, warm and moist skin, increased skin pigmentation and heat intolerance). In the hands (palmar erythema, fine tremor, sweating and clubbing). At neuromuscular levels (fatigue, atrophy, weakness, muscle fatigue and pain). At cardiovascular levels (tachycardia, palpitations, systolic hypertension and dyspnea). At psychological levels (anxiety, nervousness, irritability, insomnia, impaired concentration and reduced stress threshold) and in ocular level (bilateral exophthalmos, ptosis, periorbital edema, retraction of the upper and lower eyelid due to muscle contracture and conjunctival injection) $(2,4)$.

We find oral manifestations such as $(2,3)$ (Table 1):

\begin{tabular}{|c|c|}
\hline \multicolumn{2}{|c|}{ ORAL MANIFESTATIONS OF PATIENTS WITH THYROID GLAND DISORDERS } \\
\hline HYPERTHYROIDISM & HYPOTHYROIDISM \\
\hline 1. Accelerated dental eruption in children & 1. Delayed eruption \\
\hline 2. Maxillary or mandibular osteoporosis & 2. Enamel hypoplasia in both dentitions, (being less \\
\hline 3. Enlargement of extraglandular thyroid tissue & intense in the permanent dentition) \\
\hline (mainly in the lateral posterior tongue) & 3. Anterior open bite \\
\hline 4. Increased susceptibility to caries & 4. Macroglossia \\
\hline 5. Periodontal disease & 5. Micrognathia \\
\hline 6. Burning mouth syndrome & 6. Thick lips \\
\hline 7. Development of connective-tissue diseases like & 7. Dysgeusia \\
\hline Sjögren's syndrom or systemic lupus erythematosus & 8. Mouth breathing \\
\hline
\end{tabular}

Table 1. Oral manifestations of patients with thyroid gland disorders. 
accelerated dental eruption in children, maxillary or mandibular osteoporosis, enlargement of extraglandular thyroid tissue (mainly in lateral posterior tongue), increased susceptibility to caries and periodontal disease (possibly because these patients feel the need to consume higher quantities of sugar to meet their physical requirements), burning mouth syndrome and development of connective-tissue diseases such as Sjögren's syndrome or systemic lupus erythematosus.

Treatment of patients with thyrotoxicosis may involve antithyroid agents (propylthiouracil, carbimazole, and methimazole) which block hormone synthesis; iopanoic acid and ipodate sodium that are inhibitors of the peripheral conversion of T4 to T3; beta-blockers (propanolol) that slow the adrenergic activity and eliminate the tachycardia, anxiety, nervousness, tremors and sweating; glucocorticosteroids, such as dexamethasone, that decrease the secretion of thyroid hormone and iodine that inhibits the release of preformed hormone $(2,4)$.

- Dental management of the patient with hyperthyroidism

Before dental treatment is planned, we must carry out a detailed general clinical history, and a consultation with the specialist is recommended, to discuss the overall condition of the patient. At the time of the treatment we must consider several aspects:

1. In controlled patients, we will carry out the same dental management as in healthy patients. We must avoid severe stress situations and the spread of infectious foci (3).

2. In uncontrolled cases, we must take the same precautionary measures as in controlled patients. We must restrict the use of epinephrine or other pressor amines in local anesthetics of the retraction cords because the myocardium of these patients is sensitive to adrenaline and may unleash arrhythmias, palpitations and chest pain $(2,3,5)$. We must avoid surgical procedures because surgery, presence of acute oral infection and severe stress may precipitate thyroid storm crisis. If an emergency dental treatment is required, consultation with the patient's endocrinologist is advisable because a conservative treatment is often preferable. Treatment should be discontinued if signs or symptoms of a thyrotoxic crisis develop, and access to emergency medical services should be available. These symptoms include tachycardia, irregular pulse, sweating, hypertension, tremor, nausea, vomiting, abdominal pain and coma $(2,3)$.

3. People who have hyperthyroidism and are treated with propylthiouracil must be monitored for possible agranulocytosis, hypoproteinemia or bleeding, and a complete blood count including prothrombin time before performing any invasive procedures is usually recommended (3).

4. These patients are susceptible to central nervous sys- tem depressant drugs such as barbiturates (3).

5. In these patients proper analgesia is indicated and nonsteroidal anti-inflammatory drugs (NSAIDs) and aspirin should be used with caution (3).

- Hypothyroidism

Hypothyroidism is defined by a deficiency of the thyroid hormone. It can be acquired or by congenital defects. When it is present in infancy, it is manifested as cretinism and if it occurs in adults (especially in middle-aged women) it is known as myxedema $(3,4,6,7)$.

Characteristic signs of cretinism include mental retardation, developmental and growth delay, marked disproportion between the head and body (wide head), lack of muscle tone, overweight, less expressive face with a broad and flat nose, hypertelorism, short neck and thick, pale, dry and wrinkled skin. Myxedema is characterized by widespread metabolic slow-down, depression, overweight, diminished cardiac output and respiratory rate, decreased pulse, generalized edema (especially in face and extremities), hoarseness because the edema affects to vocal cords, sinus bradycardia, swollen nose, ears and lips, thickened and dry skin, scalp brittleness, thin or absent eyebrows and decreased sweating. Generalized edema can affect the tongue causing difficulty speaking and swallowing and serrated tongue $(3,4,6,7)$. Common oral findings in hypothyroidism include (3) (Table 1): delayed tooth eruption, enamel hypoplasia in both dentitions, being less intense in the permanent dentition, micrognathia, open bite due to lack of condylar and mandibular growth, macroglossia, thick lips, dysgeusia and mouth breathing.

Patients with hypothyroidism are treated with synthetic preparations containing sodium liothyronine, sodium levothyroixin or 1-throixine. $(3,7)$.

Hormone replacement therapy based on thyroid hormones can be prescribed in cases of severe deficiency of thyroid hormones.

- Dental management of the patient with hypothyroidism

Consulting the patients' physician and carrying out a detailed general clinical history before performing dental treatment is indicated $(3,7)$.

1. In controlled patients we must avoid oral infection $(3,7)$.

2. In uncontrolled patients, oral infection, central nervous depressants such as narcotics and barbiturates should be avoided because they may cause an exaggerated response. In controlled patients, these drugs should be used sparingly, with a reduced dosage. The presence of oral infection, central nervous depressants and surgical procedures can precipitate a myxedematous coma. Surgery procedures should also be avoided in these patients. Myxedematous coma includes hypothermia, bradycardia, severe hypotension and epileptic seizure. If that happens, dental treatment 
should be discontinued and access to emergency medical services should be available $(3,7)$.

3. Drug interactions of 1-thyroxine include (3):

- Metabolism increase using phenytoin, rifampin and carbamazepine.

- Absorption is impaired when iron sulfate, sucralfate and aluminum hydroxide are used.

- Concomitant use of tricyclic antidepressants elevates 1-thyroxine levels.

4. These patients are susceptible to cardiovascular disease, therefore they may be on anticoagulation therapy. Before dental treatment is carried out, a complete blood count is required to evaluate coagulation factors. We must avoid the use of epinephrine in local anesthetics or retraction cords. Antibiotic prophylaxis must be assessed in valvular pathology and atrial fibrillation (3).

Oral manifestations and dental management of patients with parathyroid glands disorders

Parathyroid glands secret parathyroid hormone (PTH) involved in regulating the metabolism of calcium and phosphorus. PTH plays an important role in tooth development and bone mineralization and increases bone resorption. In the kidneys, it stimulates formation of active metabolite of vitamin $\mathrm{D}$, which promotes the intestinal absorption of calcium and decreases renal reabsorption of phosphate.

\section{- Hyperparathyroidism}

Hyperparathyroidism (HPT) is characterized by hypersecretion of parathyroid hormone which occurs in three categories (8-11):

- Primary: occurs with a hyperfunction of one or more parathyroids, usually caused by a tumour (adenoma in $85 \%$ of all cases) or hyperplasia of the gland that produces an increase in PTH secretion resulting in hypercalcemia and hypophosthamia.

- Secondary: normally related to patients with intestinal malabsorption syndrome or chronic renal failure, oc- curring in a decrease of vitamin D production or with hypocalcemia causing the glands to produce a high quantity of PTH. There is also hyperphosphatemia. The PTH-related signs are brown tumors and osteitis fibrosa cystica, which is referred as renal osteodystrophy or Von Recklinghausen's disease.

- Tertiary: is an uncommon condition, affecting up to 8 $\%$ of patients with secondary HPT after a successful renal transplant. It occurs when the parathyroids activity becomes autonomous and excessive, leading to hypercalcemia (8).

The diagnosis of HPT is suspected by an increase in serum calcium and it is confirmed by the increase in PTH (12). One of the main clinical manifestations of hyperparathyroidism is bone disease. The ribs, clavicles, pelvic girdle and mandible are the bones most involved (9). In the oral cavity, the most common clinical manifestations of HPT are brown tumor, loss of bone density, weak teeth, malocclusions, soft tissue calcifications and dental abnormalities such development defects, alterations in dental eruption and widened pulp chambers (9) (Table 2).

Brown tumor presents itself as a friable red-brown mass. Its name is due to color that it takes from the haemorrhagic infiltrates and haemosiderin deposits that are often found inside. Brown tumor presents as osteolytic lesion that develops due to changes in bone metabolism caused by high serum concentration of PTH. It is mainly due to secondary HPT in patients with renal insufficiency, but it has also been described as a rare manifestation of calcium malabsorption and some forms of osteomalacia. Nowadays, brown tumor is an extremely rare manifestation of primary HPT; in these cases it is usually a result of the overproduction of the parathyroid hormone by a parathyroid tumor (single adenoma, 2 or more adenomas or carcinoma). Mandible involvement is common, especially in the area of premolars and molars, and it is rare in maxilla.

\begin{tabular}{|l|l|}
\hline \multicolumn{2}{|c|}{ ORAL MANIFESTATIONS OF PATIENTS WITH PARATHYROID GLAND DISORDERS } \\
\hline \multicolumn{1}{|c|}{ HYPERPARATHYROIDISM } & \multicolumn{1}{c|}{ HYPOPARATHYROIDISM } \\
\hline 1. Dental abnormalities: & 1. Dental abnormalities: \\
- Widened pulp chambers & - Enamel hypoplasia in horizontal lines \\
- Development defects & - Poorly calcified dentin \\
- Alterations in dental eruption & - Widened pulp chambers \\
- Weak teeth & - Dental pulp calcifications \\
- Maloclussions & - Shortened roots \\
2. Brown tumor & - Hypodontia \\
3. Loss of bone density & - Delay or cessation of dental develop- \\
4. Soft tissue calcifications & ment \\
& 2. Mandibular tori \\
& 3. Chronic candidiasis \\
& 4. Paresthesia of the tongue or lips \\
& 5. Alteration in facial muscles \\
\hline
\end{tabular}

Table 2. Oral manifestations of patient with parathyroid gland disorders. 
Radiographically, lesions are characterized as well-defined radiolucent areas, uni or multilocular. As characteristic radiographic findings we can find a widespread loss of the lamina dura, and changes in the pattern of the trabecular bone of the jaws. Long-term injures commonly produce a significant expansion of cortical, root resorption and displacement of roots can appear. Histologically, it is characterized by an abundant estroma, consisting of bundles of spindle or oval cells, and several multinucleated osteoclast-like giant cells. Calcified material can be found, as well as areas with extravastion of red blood cells and pigmentation by haemosiderin. These findings are not pathognomonic, making it necessary to perform a differential diagnosis with other lesions such as an aneurismal bone cyst, cherubism and central giant cell granuloma; being the presence of this lesion together with a history of HPT which confirms the diagnosis of brown tumor. The treatment of HPT is the first step in the management of the brown tumor, as spontaneous regression of the lesion often occurs. However, several cases of brown tumor that did not disappear or even grew after normalization of HPT level have been reported. In these cases brown tumor resection should be the preferred treatment $(8,9,13-15)$.

- Dental management of the patient with hyperparathyroidism

The clinical management of these patients does not require any special consideration. We should know that there is a higher risk of bone fracture, so we must take precaution in surgical treatments. On the other hand, it is important to recognize the presence of brown tumour and to perform a correct differential diagnosis so as not to conduct an inadequate treatment.

\section{- Hypoparathyroidism}

Hypoparathyroidism is a metabolic disorder characterized by hypocalcemia and hypophosphatemia due to a deficiency or absence of parathyroid hormone secretion. It may also develop as an isolated entity of unknown etiology (idiopathic hypoparathyroidism), or in combination with other disorders such as autoimmune diseases or developmental defects (16).

Hypoparathyroidism can cause hypocalcemia with consequent paresthesias, tetany and seizures. Disorders of ectodermal tissues are also common in these patients. These disorders include alopecia, scaling of the skin, deformities of the nails and dental abnormalities such as enamel hypoplasia in horizontal lines, poorly calcified dentin, widened pulp chambers, dental pulp calcifications, shortened roots, hypodontia and mandibular tori as PTH affects rate of eruption, formation of the matrix and calcification. A delay or cessation of dental growth and development, chronic candidiasis of the oral mucosa and nail, paresthesia of the tongue or lips and alteration of the facial muscles can occur (16) (Table 2).
This pathology is diagnosed on the basis of measurements of serum calcium and parathyroid hormone levels. The main treatments available for these patients is vitamin $\mathrm{D}$ or its analogues, calcium salts and drugs that increase renal tubular resorption of calcium, to obtain adequate, but low, normal serum calcium levels (16).

- Dental management of the patient with hypoparathyroidism

These patients have more susceptibility to caries because of dental anomalies. Dental management will be the prevention of caries with periodic reviews, advice regarding diet and oral hygiene instructions. Before performing dental treatment, serum calcium levels should be determined. They must be above $8 \mathrm{mg} / 100 \mathrm{ml}$ to prevent cardiac arrhythmias, seizures, laryngospasms or bronchospasms.

Oral manifestations and dental management of the patient with adrenal glands disorders

The adrenal glands are located on the upper pole of each kidney. They are composed of an internal or core portion (the adrenal medulla), which produce adrenaline, noradrenaline, dopamine and progesterone; and an outer portion or cortex, which is in turn responsible for the production of steroid hormones, such as: glucocorticoids (cortisol and cortisone), mineralocorticoids (aldosterone and 18-deoxycorticosterone), and androgens (dehydroepiandrosterone). The cortex has three layers: glomerular or external, where mineralocorticoids are released, the fascicular or intermediate, where glucocorticoids are produced and reticular or internal where androgens are secreted (1).

Regarding the role of substances that are secreted by the cortex, cortisol is involved in the mechanisms of adaptation of the organism to stress maintaining homeostasis; it has anti-inflammatory and immunosuppressive effect, it is responsible for mobilizing fatty acids from adipose tissue, it maintains vascular reactivity, it promotes the liver's protein synthesis via neoglycogenesis, it increases gycemia, it inhibits bone formation and delays healing.

Corticosteroid production and release from the adrenal cortex is in turn regulated by adrenocorticotropic hormone (ACTH), which is synthesized and secreted in the anterior hypophysis (adenohypophysis). In accordance to the circulating glucocorticoid concentrations, a selfregulating or negative feedback mechanism is established at hypothalamic and hypophyseal level. ACTH is in turn controlled by a series of factors such as corticotropin release hormone $(\mathrm{CRH})$, which is secreted by the hypothalamus, and by circadian rhythms (waking-sleep cycle), with cyclic variations in the plasma cortisol concentrations in the course of the 24-hour day, being maximum early in the morning and minimum at evening. Basal cortisol secretion in turn gradually increases with age, and an association moreover exists between high basal 
cortisol concentrations and a reduction in specific cognitive functions. Aldosterone is necessary for maintaining sodium and extracellular fluid balance, resulting in resorption of sodium in exchange with the potasium and hydrogen ions in the distal tubule of the nephron and in other tissues such as salivary glands and colon. It makes up to renin-angiotensin-aldosterone axis. Kidneys, in response to low blood volume, real or perceived (heart failure), secrete an enzyme called rennin, which acts in the general circulation on angiotensinogen produced by the liver and converts it into angiotensin I. Angiotensin I, under the action of the angiotensin converting enzyme (ACE), converts it in angiotensin II. Angiotensin II is a potent arteriolar vasoconstrictor and the primary regulator of aldosterone secretion, which maintains blood volume by retaining sodium (1).

- Addison's Disease

In Addison's disease or primary adrenal insufficiency exists a deficiency in the secretion of glucocorticoid and mineralocorticoid hormones by the adrenal cortex. It is associated with idiopathic, surgical, or infectious destruction or tumor of parenchyma of the adrenal gland or infiltration of the cortex by sarcoidosis, tuberculosis or amyloidosis (1).

Cortisol deficiency clinically manifests as hypoglycemia, hypotension, asthenia, muscle weakness, anorexia, nausea, weight loss and diminished resistance to infections and stress.

Characteristic melanic pigmentation may develop as a consequence of cessation of inhibition at hypophyseal level, with simultaneous increments of both ACTH and melanocyte stimulating hormone (MSH). When this happens, the skin darkens in regions such as the elbows, folds of the hands or areolas of the breasts. The oral mucosa can in turn develop black-bluish plaques, mainly affecting buccal mucosa but it can also be seen on the gums, palate, tongue and lips. The lack of aldosterone leads to sodium and liquid depletion with increased diuresis and secondary dehydration and hypotension (1).

- Dental management of the patient with Addison's disease

Most of these patients are treated with corticosteroids. We can distinguish different stages of adrenal suppression, where we can find the patient undergoing corticosteroid therapy (1):

- Stage I: doses of corticosteroids do not produce adrenal suppression.

- Stage II: the glucocorticois in blood inhibit the hypotalamic-hypophyseal-adrenal axis, and the body stops producing cortisol physiologically. This stage is therefore characterized by adrenocortical suppression, though the administered corticoid dose is still insufficient to cover the organic needs in the event of stressinducing situations.

- Stage III: the administered corticoid dose is sufficient- ly high to continue suppressing the adrenal cortex but also to cover the body needs in the event of stress.

This is interesting when we perform dental treatment because according to the stage in which the patient is, supplementation of corticosteroids may or not be necessary $(1,17)$ :

- Patients with low-dose corticotherapy (LDC) $(<30 \mathrm{mg}$ of hydrocortisone/day):

1. Patients with a history of regular corticoid use: no supplementing is required either for routine procedures, nor surgical treatments because with this dose of corticosteroid, adrenal suppresion does not occur.

2. Patients presently using corticoids: no supplementing required.

- Patients with high-dose corticotherapy (HDC) (> 40 $\mathrm{mg}$ of hydrocortisone/day):

1. Patient with a history of regular use of HDC for short periods (less than one month): the adrenal suppression is transient, recovering the stress response within 14 days after cessation of steroids. Therefore, for routine dental procedures, surgical procedures, very extensive treatments and in highly anxious patients we must consider:

Those who have discontinued corticosteroid treatment less than 14 days ago will require a daily maintenance dose on the day of treatment.

If more than 14 days: no supplementing will be required.

2. Patients with a history of regular use of HDC for more than one month: no established regimen.

3. Patients presently using HDC for one month or more: no supplementing required.

- Patients presently using 30-40 mg of hydrocortisone/ day:

If the patient is highly anxious or lengthy dental treatment or surgery procedure is to be performed, we must double the daily dose on the day of treatment. If postoperative pain is expected, we should also double the daily dose on the first postoperative day.

- Patients receiving corticotherapy on alternate days for at least 30 days:

On the non-corticoid days, no supplementing is required. Conservative management is indicated on the rest of the days.

- Patients with topical or inhaled steroids: no supplementing required.

Other aspects of dental management of these patients are (1):

1.Conducting treatment in the morning.

2. Control of anxiety and emotional stress.

3. Use long-acting anesthetics.

4. Treatment of postoperative pain.

5. Prevention of iatrogenic fracture during surgery in patients with a long history of continuous corticotherapy, 
because glucocorticoids can increase the risk of fracture by causing osteoporosis.

6. Consideration is also required of the possible interactions of glucocorticoids with a other drugs:

- Phenytoin, barbiturates and rifampicin accelerate glucocorticoid metabolism.

- Prednisone bioavailability decreases with the administration of antacids.

- Glucocorticoids increase the requirements of insuline, oral antidiabetic drugs and hypotensive medication.

\section{- Addisonian Crises}

Addisonian crises or acute adrenococortical insufficiency is a rare but serious complication in patients with primary Addison's disease. Although, actually it is more likely to be attributable to secondary adrenal failure (administration of high-doses of exogenous corticosteroids therapeutically) than to Addison's disease. The reason for this is the sudden withdrawal of exogenous corticoids, or the existence of situations requiring greater amounts of corticoids than those afforded by replacement therapy. It presents as a sudden failure of the adrenal cortex function. The resulting clinical picture compromises shock with nausea, vomiting, abdominal pain and hypotension. Fever and hypothermia may be observed and can lead to coma and death (1).

- Dental management of the patient with Addisonian crises

Prevention is the best management approach for Addisonian crises. We should perform a detailed clinical history and a consultation with the specialist is recommended, and we take action accordingly. However, if crisis takes place, we should interrupt dental procedure, place the patient in dorsal decubitus and contact with the corresponding medical emergency service. Until medical help arrives, the patient should be administered oxygen (5-10 liters/min). If the patient is unconscious, he should be placed in dorsal decubitus with the legs raised, and we should notify the emergency service to arrange transfer of the patient to the hospital. Before four minutes have elapsed, basic vital support should be provided in accordance to the patient's condition. If an adrenal cause is suspected, $100 \mathrm{mg}$ of hydrocortisone should be administered intravenously or intramuscularly, within 30 seconds if possible, and two hours later, another $100 \mathrm{mg}$ of hydrocortisone dissolved in saline for intravenous or intramuscular injection should be provided (1).

- Hyperadrenocorticism (Cushing's syndrome)

Cushing's syndrome (CS) refers to manifestations induced by chronic exposure to excess glucocorticoids produced by the adrenal cortex. This excess can be caused by various reasons. It most commonly arises from iatrogenic causes (due to administration of exogenous glucocorticoids). The second most common cause is a high production of ACTH as a result, in most cases, of the presence of a pituitary corticotroph adenoma or less frequently by an extrapituitary tumor or very rarely by a tumor secreting CRH. $(18,19)$.

CS is suspected in the presence of central obesity with supraclavicular fat accumulation and a cervical fat pad, moon face, thinned skin, acne and hirsutism. Hypertension, glucose intolerance, menstrual irregularity, osteoporosis and pathological fractures, delayed healing, increased risk of infection and neuropsychological disturbances including depression, emotional irritability, sleep disturbances and cognitive deficits are also observed. Purple striae and muscular atrophy are particularly positive stigmata in adults, whereas in child growth retardation are frequently present $(18,19)$.

- Dental management of the patient with hyperadrenocorticism

Dental management in these patients consists in prevention of infections, pathological fractures during surgical treatments and complications such as hypertension, hyperglycemia, depression and delayed healing. In patients on steroids we must evaluate the need to administer additional corticosteroids.

\section{Discussion}

1. More common oral manifestations in patients with endocrine disorders are:

- Thyroid glands: in hyperthyroidism: accelerated dental eruption, osteoporosis, enlargement of extraglandular thyroid tissue, caries and periodontal disease, burning mouth syndrome and development of connective-tissue. In hypothyroidism: delayed eruption, enamel hypoplasia, micrognathia, open bite, macroglossia, thick lips, dysgeusia and mouth breathing.

- Parathyroid glands: in HPT: brown tumor, loss of bone density, soft tissue calcification and dental abnormalities; in hypoparathyroidism: dental abnormalities, a delay or cessation of dental growth and development, paresthesia of the tongue or lips and alteration of the facial muscles.

- Adrenal glands: in Addison's disease: black-bluish plaques in buccal mucosa.

2. Dental management of patients with endocrine disorders:

- Thyroid glands: in hyperthyroidism: We must restrict the use of adrenaline and avoid severe stress situations and the spread of infectious foci. In patients treated with propylthiouracil, a complete blood count is usually recommended. NSAIDs and aspirin should be used with caution. Treatment should be discontinued if signs or symptoms of a thyrotoxic crisis develop; in hypothyroidism: a complete blood count before performing dental treatment is recommended. We must restrict the use of adrenaline and avoid oral infection and central nervous depressant drugs. We should know the drug interactions of 1-thyroxine. If myxedematous coma develops, we will only carry out emergency dental treatment. 
- Parathyroid glands: in HPT: there is a higher risk of bone fracture; in hypothyroidism: control the development of caries.

- Adrenal glands: in Addison's disease: We must conduct treatment in the morning and control of anxiety. In patients with corticotherapy, we must evaluate the necessity to administer additional corticosteroids; in Addisonian crises: we should interrupt dental procedure; in hyperadrenocorcism: we must prevent infection and pathological fractures, and we must evaluate the necessity to administer additional corticosteroids.

\section{References}

1. Lorenzo-Calabria J, Grau D, Silvestre FJ, Hernández-Mijares A. Management of patients with adrenocortical insufficiency in the dental clinic. Med Oral. 2003;8:207-14.

2. Little JW. Thyroid Disorders. Part I: Hyperthyroidism. Oral Surg Oral Med Oral Pathol Oral Radiol Endod. 2006;101:276-84.

3. Pinto A, Glick M. Management of patients with thyroid disease: oral health considerations. J Am Dent Assoc. 2002;133:849-58.

4. Woeber KA. Update on the management of hyperthyroidism and hypothyroidism. Arch Fam Med. 2000;9:743-7.

5. Yagiela JA. Adverse drug interactions in dental practice: interactions associated with vasoconstrictors. Part V of a series. J Am Dent Assoc. 1999;130:701-9.

6. Roberts CG, Ladenson PW. Hypothyroidism. Lancet. 2004;363:793803.

7. Little JW. Thyroid disorders. Part II: hypothyroidism and thyroiditis. Oral Surg Oral Med Oral Pathol Oral Radiol Endod. 2006;102:14853.

8 Magalhães DP, Osterne RL, Alves AP, Santos PS, Lima RB, Sousa FB. Multiple brown tumours of tertiary hyperparathyroidism in a renal transplant recipient: a case report. Med Oral Patol Oral Cir Bucal. 2009;15:e10-3.

9. Triantafillidou K, Zouloumis L, Karakinaris G, Kalimeras E, Iordanidis F. Brown tumors of the jaws associated with primary or secondary hyperparathyroidism. A clinical study and review of the literature. Am J Otolaryngol. 2006;27:281-6.

10. Hougardy DM, Peterson GM, Bleasel MD, Randall CT. Is enough attention being given to the adverse effects of corticosteroid therapy? J Clin Pharm Ther. 2000;25:227-34.

12. Sutbeyaz Y, Yoruk O, Bilen H, Gursan N. Primary hyperparathyroidism presenting as a palatal and mandibular brown tumor. J Craniofac Surg. 2009;20:2101-4.

13. Padbury AD Jr, Tözüm TF, Taba M Jr, Ealba EL, West BT, Burney $\mathrm{RE}$, et al. The impact of primary hyperparathyroidism on the oral cavity. J Clin Endocrinol Metab. 2006;91:3439-45.

14. Fernández-Sanromán J, Antón-Badiola IM, Costas-López A. Brown tumor of the mandible as first manifestation of primary hyperparathyroidism: diagnosis and treatment. Med Oral Patol Oral Cir Bucal. 2005;10:169-72.

15. Martínez-Gavidia EM, Bagán JV, Milián-Masanet MA, Lloria de Miguel E, Pérez-Vallés A. Highly aggressive brown tumour of the maxilla as first manifestation of primary hyperparathyroidism. Int $\mathrm{J}$ Oral Maxillofac Surg. 2000;29:447-9.

16. Yamazaki H, Ota Y, Aoki T, Karakida K. Brown tumor of the maxilla and mandible: progressive mandibular brown tumor after removal of parathyroid adenoma. J Oral Maxillofac Surg. 2003;61:719-22.

17. Kelly A, Pomarico L, de Souza IP. Cessation of dental development in a child with idiopathic hypoparathyroidism: a 5-year follow-up. Oral Surg Oral Med Oral Pathol Oral Radiol Endod. 2009;107:673-7.

18. Gibson N, Ferguson JW. Steroid cover for dental patients on longterm steroid medication: proposed clinical guidelines based upon a critical review of the literature. Br Dent J. 2004;197:681-5.

19. Bertagna X, Guignat L, Groussin L, Bertherat J.Cushing's disease. Best Pract Res Clin Endocrinol Metab. 2009;23:607-23.
20. Arnaldi G, Angeli A, Atkinson AB, Bertagna X, Cavagnini F, Chrousos GP, et al. Diagnosis and complications of Cushing's syndrome: a consensus statement. J Clin Endocrinol Metab. 2003;88:5593602 . 